\title{
Contact YAG Laser Palatopharygoplasty
}

\author{
Hwoe Young Ann
}

Department of Otolaryngology, College of Medicine Kyung Hee University, Seoul, Korea

Snoring, the lay term for obstructive breathing during sleep, is one of the most prevalent of obnoxious human habits. In a 30 - to 35 -year-old population, $20 \%$ of men and $5 \%$ of women will snore; by age $60,60 \%$ of men, and $40 \%$ of women will snore habitually.

Most of the heavy snorer with or without sleep apnea have symtoms of 1) loud, irregular snoring, and snorts, gasps and other unusual brething sounds during sleep, 3) pauses in breathing during sleep, 4) excessive day time sleepiness and 5) fatigue, 6) obesity, 7) changes in olertness, memory, personality, or behavior, 8) impotence, 9) morning headache, and 10) bed wetting.

Common anatomical factors contributuing to snoring are 1) imcompetent tone of palatal, pharyngeal, and giossal muscles, 2) space occupying massaes 3 ) excessive length of the soft palate and uvula, and 4) obstructive nasal breathing.

Various metnods have been utilized in order to improve snoring and/or obstructive sleep apnea syndrome; weight reduction, change of sleep positon, stop or reduce alcohol or sedaives, some medications, nasal continuous positive airway pressure (CPAP). and uvulopalatopharyngoplasty (UPPP). The surgical procedure, UPPP has been known the most effective method, with very satisfactory result; elimination or improvement of snoring was reported by Pelausa in $75.9 \%$ of patients and by Chouard in $86.3 \%$ of patients.

But the converntional UPPP has disadvantages of general anesthesia, several days of hospital stay, severe post-operative pain and high cost for the patients. So, couple of years ago, Dr. Kamami Yves-Victor of Foch Hospital in Paris introduced the $\mathrm{CO}_{2}$ laser resection of the palatopharynx (LRPP).

LRPP of Dr. Kamami was a new technique, safe and effective, ambulatory by local anesthesia, performed for the treatment of snoring and and in somr cases of obstructive sleep apnea syndrome(OSAS). The LRPP was based on the progressive inlargement of the air space in the oropharynx, to eliminate or reduce obstructions that occur during sleep, by successive "vaporizations" of the vibrating soft palate, wide posterior tonsil pillars and redundant posterior pharyngeal mucosa. According to Dr. Kamami, he had the $85 \%$ of success rate, and Dr. Jack A. 
Coleman, Vanderbilt University, Who learned this technique from Kamami and introduced it to U.S., produced $85-95 \%$ of success rate.

But this tecnique LRPP with $\mathrm{CO}_{2}$ laser needs 3 to 7 operations, usually 3 to 5 for the women, 4 to 5 for the men, "non-OSAS", interval with a fornight to 3 or 4 weeks berween each. That means 4 to 8 months are necessary until the prodedures are completed, although the procedures are simple, reliable, non-invasive and painless procedure under local anesthesia without hospitalization and also operator must prepare the new special type of handpiece to protect the posterior pharyngeal wall from $\mathrm{CO}_{2}$ laser beam.

So I tried mre simple, one stage operation with same effectiveness, using the contact YAG laser. Under the local anesthesia with $10 \%$ lidocaine spray into the oropharynx first, then injection of $2 \%$ lidocaine with epinephrine, I began to resect the part of posterior pillar, soft palate and uvula, using 7-9 watt of contact YAG taser. After excision of redundant soft tissue of the soft palate and pharynx. I sutured the antarior and posterior resected margins of the mucosa with 4-0 vicryl. After the procedure I gave analgesics and antibiotics to the patient.

Compare to $\mathrm{CO}_{2}$ LRPP, my contact YAG LPPP was safer, more simple, more convenient, easier to handle, and cheaper economical procedure.

I have done 10 to 15 procedures each since last year. Usually, about $8.3 \%$ of patients were safisfied with this procedure. That means elimination or improvement of snoring.

Side effects were post-operative pain and nasal regurgitation. Post-operative swallowing pain was followed 5 to 7 days, but was well controlled by analgesics medication and $2 \%$ lidocaine biscous gargling solution. Mild nasal regurgitaion was complained in first week in some patients, but improved spontaneouly in most patients. There were no patients who complain the change of tone of voice. 